\title{
Large coronary pseudoaneurysm with pulmonary artery fistula, six months after left main trunk stenting with paclitaxel-eluting stent
}

\author{
Alexandru MT Hagau', Sorin M Dudea ${ }^{2}$, Radu Hagau ${ }^{3}$, Natalia Hagau ${ }^{4}$ \\ ${ }^{1}$ Deparment of Cardiology, "Niculaie Stancioiu” Heart Institute Cluj-Napoca, Romania \\ 2 Department of Radiology, "Iuliu Hatieganu" University of Medicine and Pharmacy Cluj-Napoca, Romania \\ ${ }^{3}$ St. Elizabeth's Medical Center, Boston, Massachusetts, USA \\ ${ }^{4}$ Department of Anaesthesia"Iuliu Hatieganu" University of Medicine and Pharmacy Cluj-Napoca, Romania
}

\begin{abstract}
There are no reported cases of a pseudoaneurysm leading to a fistula into the pulmonary artery after percutaneous coronary intervention. We describe a patient who developed a late pseudoaneurysm after left main trunk (LMT) stenting with paclitaxeleluting stent, discovered during coronary angiography. Transesophageal echocardiography and contrast-enhanced computed tomographic scan revealed a pericardial effusion, a large pseudoaneurysm communicating with LMT and a fistula into the pulmonary artery. The recommended therapy was surgery.
\end{abstract}

Keywords: percutaneous coronary intervention, fistula, coronary pseudoaneurysm

\section{Introduction}

Pseudoaneurysms (PsA) of the coronary arteries are described less commonly than aneurysms. They usually appear after catheter-based coronary interventions as a result of traumatic dissection or perforation of a coronary artery, resulting in an interruption of one or more of the three vessel wall layers [1-3].

Coronary intervention-associated PsA are mainly discovered during subsequent angiography when patients present with recurrent angina [3]. Based on several case reports, they have been associated with an adverse outcome if untreated [1]. There are only a few reported cases of PsA arising from the left main coronary ostial button (acquired late after aortic root replacement) and leading to a fistula into the pulmonary artery [4-6].

Received 20.11.2012 Accepted 15.12.2012

Med Ultrason

2013, Vol. 15, No 1, 59-62

Corresponding author: Alexandru MT Hagau, M.D.

Deparment of Cardiology

"Niculaie Stancioiu" Heart Institute Cluj-Napoca 400001 Motilor 19-21, Cluj-Napoca, Romania

Fax/Tel +40 746055989

e-mail: daduhagau@yahoo.com

\section{Case report}

A 68 year old man was admitted in our hospital with unstable angina (CCS 4). His medical history was significant for arterial hypertension, diabetes mellitus, dyslipidemia, obesity, coronary artery disease, permanent atrial fibrillation, and peripheral vascular disease. Current cardiac medication consisted of nitrates, beta-adrenergic receptor blockers, statine, calcium channel blockers, angiotensine converting enzyme inhibitor, dicumarine, and aspirine. During the episode of chest pain he presented ST depression in V1 to V3 leads.

Coronary angiography was performed and revealed $40 \%$ stenosis of the left main shaft, $70 \%$ stenosis of the middle right coronary artery (RCA), $70 \%$ stenosis of the proximal left anterior descending artery (LAD) and a diffusely decreased left circumflex artery (LCX) around $1.7 \mathrm{~mm}$ diameter. The percutaneous coronary intervention was performed and a $3 \mathrm{~mm}$ x $20 \mathrm{~mm}$ bare metal stent (BMS) Presillion (Cordis) was placed on the middle RCA, with good result: $90 \%$ lumen diameter, TIMI (Thrombolysis in Myocardial Infarction) 3 flow. A second $3 \mathrm{~mm}$ x $24 \mathrm{~mm}$ bare metal stent Arthos (AMG International) was placed on proximal LAD resulting in $100 \%$ lumen diameter with TIMI 3 flow (fig 1a). 

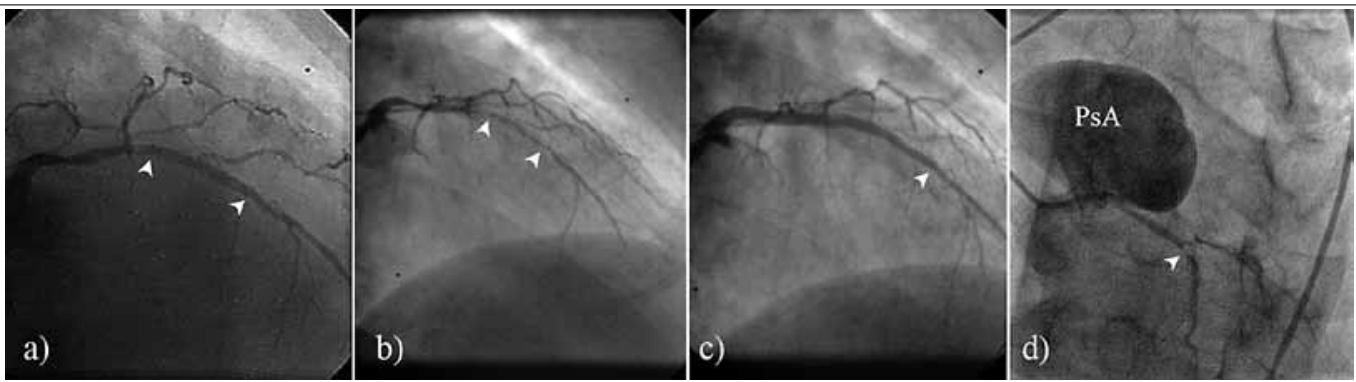

Fig 1. a) Left coronary angiography showing on right anterior oblique cranial projection a $3 \mathrm{~mm} \times 24 \mathrm{~mm}$ bare metal stent on proximal left anterior descending artery (two arrowhead point the stent), the first percutaneous coronary intervention; b) Left coronary angiography showing, on right anterior oblique with cranial projection, diffuse left anterior descending artery (LAD) in stent restenosis (two arrowhead point the stent), $90 \%$ ostial LAD stenosis, $60 \%$ left main shaft stenosis, 7 weeks later; c) Left coronary angiography showing, on right anterior oblique with cranial projection, two paclitaxel eluting stents: first $3,5 \mathrm{~mm}$ $\mathrm{x} 28 \mathrm{~mm}$ PES on left main trunk overlapping the second $3 \mathrm{~mm}$ x $32 \mathrm{~mm}$ PES on LAD which cover the implanted BMS, the arrowhead points the distal end of the PES stent on LAD; d) Left coronary angiography showing, on left anterior oblique with cranial projection, the pseudoaneurysm (PsA) above LMT and arrowhead points the focal restenosis on ostial LAD.
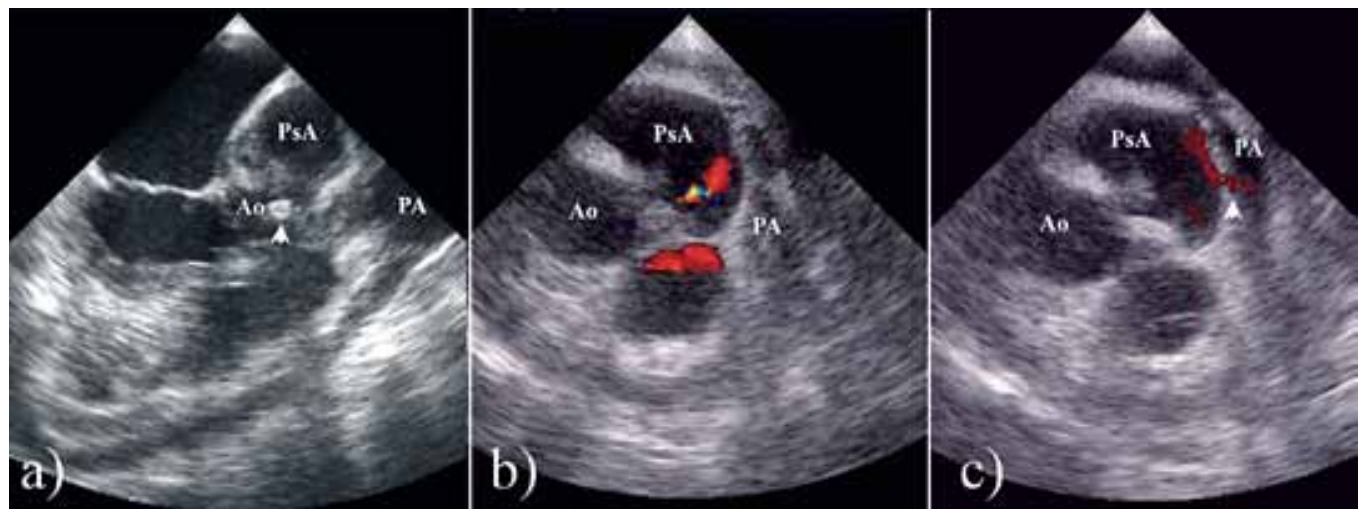

Fig 2. Transesophageal echocardiography: a) mid esophageal location showing the pseudoaneurysm (PsA) after left main trunk PES stenting, the arrowhead points the stent; b) superior esophageal location showing the communication between pseudoaneurysm (PsA) and stented LMT with restricted flow on color Doppler; c) superior esophageal location- the arrowhead points the communication between the pseudoaneurysm (PsA) and pulmonary artery (PA) with flow on color Doppler.

Seven weeks later the patient was readmitted for rest angina. Coronary angiography showed diffuse severe LAD in stent restenosis, $90 \%$ ostial LAD stenosis and $60 \%$ left main shaft stenosis (fig $1 \mathrm{~b}$ ). A second PCI was performed using drug eluting stent (DES). A guiding catheter Launcher EBU 3.5 SH 7Fr (Medtronic) was used. Two coronary guide wires, Hi-Torque Balance Middleweight $0.014 \% / 190 \mathrm{~cm}$ (Abbott Vascular), were inserted in the LAD and LCX. The predilatation of the proximal LAD with a $2 \mathrm{~mm} \times 20 \mathrm{~mm}$ Fire Star (Cordis) balloon catheter (12atm) was done. A 3,5mm x 28mm Taxus Liberte (Boston Scientific) Paclitaxel Eluting Stent (PES) was placed on left main from the ostium up to $2 \mathrm{~mm}$ to the bifurcation. The PES was deployed with 11atm. Overlapping this stent a $3 \mathrm{~mm}$ x $32 \mathrm{~mm}$ Taxus Liberte second PES was placed ending distally to the LAD BMS. Left main and the overlapped segment of the two PESs were dilated using a $3,5 \mathrm{~mm}$ x $15 \mathrm{~mm}$ Dura Star (Cordis) noncompliant balloon with $15 \mathrm{~atm}$, resulting TIMI 3 flow and 100\% lumen diameter (fig 1c). Intravascular ultrasonography (IVUS) was not performed.

It was the third hospital admission after 6 months for rest angina when coronary angiography revealed $70 \%$ ostial LAD restenosis and 50\% restenosis in the distal segment of the LAD PES stent. In addition a $6 \mathrm{~cm}$ x $4 \mathrm{~cm}$ 
ovoid cavity (PsA) originating above the ostium of the left main trunk was found (fig 1d).

Transesophageal echocardiography (TEE) showed a pericardial (no tamponade) effusion and a large paraaortic PsA, extending to the pulmonary artery trunk (fig 2a). This PsA communicates with LMT by a $4 \mathrm{~mm}$ orifice with restricted flow on Doppler (fig $2 b$ ). Surprisingly, a communication between the PsA and the pulmonary artery was found on TEE in the superior esophageal position (fig 2c).

An additional 64-slice contrast-enhanced computed tomographic scan of the chest confirmed the presence of the pericardial effusion, PsA and fistula between the PsA and the pulmonary artery (fig 3 ) and allowed the relationship of the PsA to its surrounding structures.

The recommended therapy was surgery: CABG with resection of the PsA and closure of the pulmonary artery fistula.

\section{Discussion}

LMT post procedural PsA formation is very rare. There are no reported cases of post procedural PsA leading to a fistula into the pulmonary artery.

In our case the diagnosis has proven challenging, we discovered a large PsA during coronary angiography when patient presented recurrent angina. We found the precise location and the connection between the stented LMT and the PsA but we did not know about fistula. In addition, TEE examination allowed identification and assessment of the fistulous connection to pulmonary artery and the presence of pericardial effusion. Some previous reports advocate computed tomography as a diagnostic tool [4]. The computed tomographic scan allowed assessment of size and relationship of the PsA to its surrounding structures and confirmed the presence of fistula.

In the present case the PsA developed following coronary laceration during PES implantation, without blood seepage through the adventicia. This might be a result of dissection that was not successfully sealed because of incomplete stent expansion over the dissected site. The IVUS was not used but there was $100 \%$ LMT lumen diameter and TIMI 3 flow at the final angiographic procedure. The development of a late PsA can be explained by the inhibition of the healing process due to PES. Small dimension lacerations led to noncompresive pericardial effusion. Progressive PsA was formed separately of pericardial effusion. Pulmonary artery fistula was a result of the high pressure carried out by the PsA.

There is no standard therapy recommended for PsA occurring after coronary interventions [7]. PsA occurring

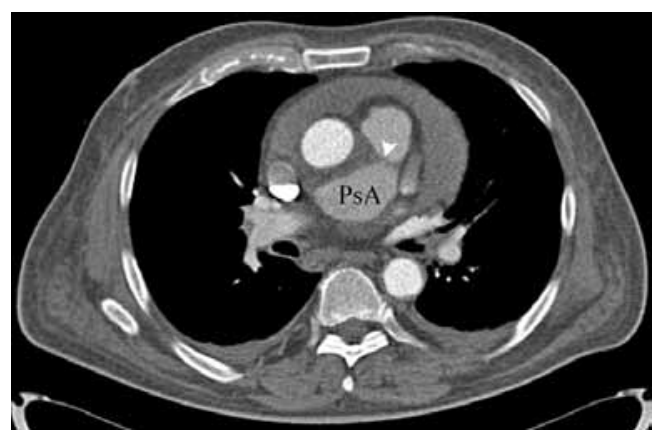

Fig 3. Axial computed tomographic view of pseudoaneurysm (PsA) with connection to pulmonary artery (PA).The arrowhead points the fistula.

slowly after intervention have been successfully treated with IVUS guided covered stenting, some patients were untreated and the PsA closed spontaneously, others underwent CABG with resection of the PsA, or had the pesudoaneurysm closed by spring coil embolization [7]. For our patient, having a large postinterventional PsA with pulmonary fistula, the recommended therapy was surgery.

\section{Conclusion}

Coronary intervention-associated PsA was discovered during subsequent angiography. An extra TEE examination and computed tomography scan allowed identification and assessment of the fistulous connection to pulmonary artery and the presence of pericardial effusion, and a clearer diagnosis of complications.

\section{References}

1. Maehara A, Mintz GS, Castagna MT, et al. Intravascular ultrasound assessment of spontaneous coronary artery dissection. Am J Cardiol 2002; 89: 466-468.

2. Kitzis I, Kornowski R, Miller HI. Delayed development of a pseudoaneurysm in the left circumflex artery following angioplasty and stent placement, treated with intravascular ultrasound-guided stenting. Cathet Cardiovasc Diagn 1997; 42: 51-53.

3. Schobel WA, Voelker W, Haase KK, Karsch KR. Occurrence of a saccular pseudoaneurysm formation two weeks after perforation of the left anterior descending coronary artery during balloon angioplasty in acute myocardial infarction. Cathet Cardiovase Interv 1999; 47: 341-346.

4. Ueno M, Imada T, Nonaka K, Oda T. Aortopulmonary fistula after aortic root replacement. Ann Thorac Surg 2002; 74: 590-591. 
62 Alexandru MT Hagau et al

5. Chevalier P, Moncada E, Kirkorian G, Touboul P. Acquired aortopulmonary fistula in pseudoaneurysm of the aorta six years after a Bentall operation. J Thorac Cardiovasc Surg 1995; 110: 1143-1144.

6. Ibe R, Bahktiari N, Davidson C, Hildick-Smith D, Lewis M. Large aortic pseudoaneurysm, from left coronary os-
Large coronary pseudoaneurysm with pulmonary artery fistula

tium, with aortopulmonary fistula 10 years after aortic root replacement for type A aortic dissection. Circulation 2011; 123: e580-e582.

7. Aqel RA, Zoghbi GJ, Iskandrian A. Spontaneous coronary artery dissection, aneurysms, and pseudoaneurysms: a review. Echocardiography 2004; 21: 175-182. 